\title{
Outer divertor of ASDEX Upgrade in low-density L-mode discharges in forward and reversed magnetic field \\ Part II: Analysis of local impurity migration
}

\author{
L. Aho-Mantila ${ }^{1}$, M. Wischmeier ${ }^{2}$, K. Krieger ${ }^{2}$, V. Rohde ${ }^{2}$, \\ A. Hakola ${ }^{1}$, S. Potzel ${ }^{2}$, A. Kirschner ${ }^{3}$, D. Borodin ${ }^{3}$, \\ ASDEX Upgrade Team \\ 1 VTT Technical Research Centre of Finland, P.O.Box 1000, FI-02044 VTT, \\ Finland \\ 2 Max-Planck-Institut für Plasmaphysik, D-85748 Garching, Germany \\ 3 Institute of Energy and Climate Research - Plasma Physics, \\ Forschungszentrum Jülich GmbH, Association EURATOM-FZJ, Partner in the \\ Trilateral Euregio Cluster, Jülich, Germany \\ E-mail: leena.aho-mantila@vtt.fi
}

\begin{abstract}
Part I presented a detailed analysis of outer divertor plasma conditions in low-density L-mode discharges in ASDEX Upgrade. In this paper, we analyse the local migration of carbon that originates from ${ }^{13} \mathrm{CH}_{4}$ injected into these plasmas from the vertical outer target. Notable changes are observed in the local carbon deposition patterns when reversing the magnetic field in the experiments. Kinetic impurity-following simulations are performed using the 3D ERO code package with $2 \mathrm{D}$ background plasma solutions calculated with the SOLPS5.0 code package. The modelling shows that the measured changes are due to the changes in plasma collisionality, dissociation and ionization rates, and $\mathbf{E} \times \mathbf{B}$ drift of the impurities. These conditions affect the direction and rate of impurity migration inside and out of the divertor, having wider consequences on the global migration of impurities in a divertor tokamak. It is further shown, that the migration pathways are largely determined by carbon ions and, hence, relevant for impurities in general. Neutral carbon and hydrocarbons are deposited only in the near vicinity of the injection, where they affect the local re-deposition efficiency. In this limited region, a perturbation of the local plasma conditions by the methane puff appears likely, yielding a significant uncertainty for interpreting the deposition efficiencies. The local deposition is largely influenced by the magnetic presheath electric field, the structure of which is the main uncertainty in the SOLPS5.0-ERO simulations.
\end{abstract}

Submitted to: Nuclear Fusion 


\section{Introduction}

The outer divertor is subject to substantial plasma-wall interaction processes, which erode the target material, releasing impurities into the divertor plasma. These impurities may become transported into the core, where they dilute and cool the fuel, or migrate onto other wall segments or remote areas, changing the surface material composition or leading to dust accumulation. Conversely, material eroded from the main chamber wall can also accumulate in the outer divertor, depending on the migration properties of impurities [1,2]. The consequences may be critical for the operation of future fusion devices with high duty cycle, for which reason material selection has been one of the key design issues for ITER [3], and will be even more crucial for the design of a fusion reactor [2]. In this paper, we analyse the local redeposition and migration of impurities in the ITER-relevant, vertical outer target of ASDEX Upgrade (AUG).

The analysis is based on low-density L-mode experiments performed during the AUG campaigns in 2007-2009. During this period, all plasma-facing components in AUG were coated with tungsten. Part I presented a thorough discussion of the plasma properties in these experiments. In this paper, we focus on ${ }^{13} \mathrm{CH}_{4}$ injected from the outer target during repeated discharges at the end of the experimental campaigns. The impurity injections were monitored in-situ using spectroscopy and post-mortem using ion beam techniques for the tiles surrounding the injection locations $[4,5]$. Well-resolved 2-dimensional re-deposition patterns of ${ }^{13} \mathrm{C}$ were obtained, reflecting the detailed migration mechanisms taking place in the vicinity of the surface. It is observed that the impurities have notably different migration behaviour when the toroidal magnetic field, $B_{\mathrm{T}}$, and plasma current, $I_{\mathrm{p}}$, are reversed in the experiments.

Following the experimental analysis, we present a detailed interpretation of local impurity migration using ERO simulations. ERO is an impurity following code that calculates the kinetic 3-dimensional trajectories of trace impurities in a static plasma background, taking into account re-deposition and re-erosion processes at the material surface [6]. The SOLPS5.0 solutions derived in Part I are used for describing 2D plasma backgrounds for the ERO simulations.

The paper is organized as follows. Section 2 summarizes the experimental results from local AUG ${ }^{13} \mathrm{CH}_{4}$ injection experiments in 2007-2009. In section 3, the migration of carbon is analysed in ERO simulations. Conclusions are presented in section 4.

\section{Experimental results from local ${ }^{13} \mathrm{C}$ injection experiments in 2007-2009}

To investigate the local migration of carbon in an ITER-relevant vertical target geometry, ${ }^{13} \mathrm{CH}_{4}$ was injected into the outer divertor of AUG in three low-density L-mode experiments summarized in table 1 . The first two experiments were made in forward field (ion $\nabla \mathbf{B}$ drift towards the lower divertor) and had similar plasma conditions, particularly in the outer divertor. As described in Part I, the outer divertor was in a low-recycling regime, with $25 \mathrm{eV}$ peak target temperature and $1 \times 10^{19} \mathrm{~m}^{-3}$ peak target density. In the reference experiment, denoted $\mathrm{Ex}_{\mathrm{fwd}},{ }^{13} \mathrm{CH}_{4}$ was injected from two poloidal locations into the SOL. In the second experiment, denoted $\mathrm{Ex}_{\mathrm{src}}$, only the upper poloidal location was used, and the strike point was shifted $3 \mathrm{~cm}$ upwards, see figure 1. This means that the puffing was made into a different location in the SOL plasma compared to the two injections in $\mathrm{Ex}_{\mathrm{fwd}}$. The primary motivation for performing $\mathrm{Ex}_{\mathrm{src}}$ originated from the recent discussion of strong impurity puffs 
Table 1. Summary of experimental conditions. Discharges in parentheses are characterization discharges without ${ }^{13} \mathrm{CH}_{4}$ injection.

\begin{tabular}{|c|c|c|c|c|c|c|c|}
\hline Exp. & year & discharges & $\begin{array}{l}B_{\mathrm{T}} \\
(\mathrm{T})\end{array}$ & $\begin{array}{c}I_{\mathrm{p}} \\
(\mathrm{MA})\end{array}$ & $\begin{array}{c}\bar{n}_{\mathrm{e}} \\
\left(\times 10^{19} \mathrm{~m}^{-3}\right)\end{array}$ & $\begin{array}{l}P_{\mathrm{ECRH}} \\
(\mathrm{MW})\end{array}$ & $\begin{array}{l}P_{\text {Ohmic }} \\
(\mathrm{MW})\end{array}$ \\
\hline $\operatorname{Ex}_{\mathrm{fwd}}$ & 2007 & $\begin{array}{l}(\# 22469), \# 22573-5, \\
\# 22577-81, \# 22583-5\end{array}$ & -2.5 & 0.8 & 3.2 & 0.60 & 0.35 \\
\hline $\mathrm{Ex}_{\mathrm{src}}$ & 2008 & $\begin{array}{l}(\# 24178), \# 24183 \\
\# 24187-9\end{array}$ & -2.5 & 0.8 & 3.2 & 0.65 & 0.30 \\
\hline $\mathrm{Ex}_{\mathrm{rev}}$ & 2009 & $(\# 25881), \# 25883-90^{*}$ & 2.5 & -0.8 & 3.4 & 0.90 & 0.30 \\
\hline
\end{tabular}

${ }^{*}$ no ${ }^{13} \mathrm{CH}_{4}$ puff in \#25889

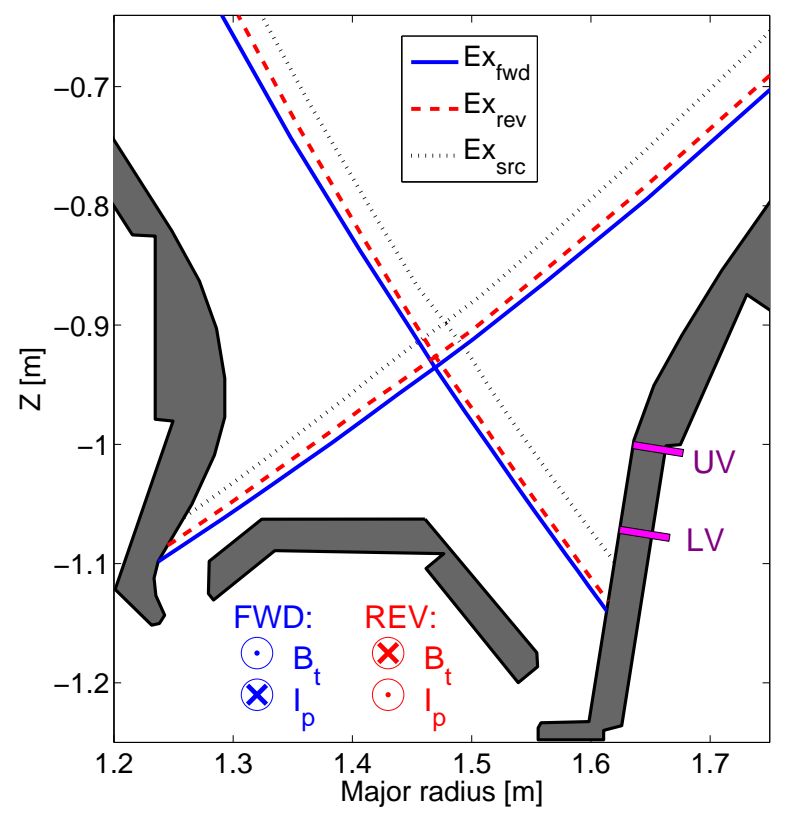

Figure 1. Steady-state separatrix configurations in the three experiments $\mathrm{Ex}_{\mathrm{fwd}}$, $E x_{\text {src }}$, and $E x_{\text {rev }}$. The locations of the two divertor valves (UV, LV) for the ${ }^{13} \mathrm{CH}_{4}$ injection are shown in magenta. The directions of $B_{\mathrm{T}}$ and $I_{\mathrm{p}}$ in the forward and reversed field configurations are indicated at the bottom.

possibly perturbing the local plasma conditions [7], for which reason the injection rate in $\mathrm{Ex}_{\mathrm{src}}$ was reduced compared to $\mathrm{Ex}_{\mathrm{fwd}}$. The number of discharges was 11 in $\mathrm{Ex}_{\mathrm{fwd}}$ and 4 in $\mathrm{Ex}_{\mathrm{src}}$. The third experiment, denoted $\mathrm{Ex}_{\mathrm{rev}}$, was performed in reversed field. The magnetic geometry and main plasma parameters were kept as similar as possible to $\mathrm{Ex}_{\mathrm{fwd}}$, with the exception of external heating power that was increased from 0.6 MW to $0.9 \mathrm{MW}$ to ensure well-attached outer divertor plasma. Two injection locations were used in 8 discharges, with similar injection rates and total injected amounts as in $\mathrm{Ex}_{\mathrm{fwd}}$.

In the following, we summarize the experimental results obtained in these studies. The deposition efficiencies reported earlier for $\mathrm{Ex}_{\mathrm{fwd}}[4]$ and $\mathrm{Ex}_{\mathrm{rev}}[5]$ are recalculated 
Table 2. ${ }^{13} \mathrm{CH}_{4}$ injection locations $\left(\mathrm{d} S=S-S_{\mathrm{sep}}\right)$ and measured local plasma parameters (see Part I). The net local re-deposition efficiencies are determined by dividing the measured number of locally deposited ${ }^{13} \mathrm{C}$ atoms by the total injected number of ${ }^{13} \mathrm{CH}_{4}$ molecules.

\begin{tabular}{lcccccccc}
\hline Exp. & valve & $\begin{array}{c}\mathrm{d} S \\
(\mathrm{~cm})\end{array}$ & $\begin{array}{c}T_{\mathrm{e}} \\
(\mathrm{eV})\end{array}$ & $\begin{array}{c}n_{\mathrm{e}} \\
\left(\times 10^{18}\right)\end{array}$ & $\begin{array}{c}\text { puff rate } \\
\left(\times 10^{19} 1 / \mathrm{s}\right)\end{array}$ & $\begin{array}{c}\text { injected } \\
\left(\times 10^{20}\right)\end{array}$ & $\begin{array}{c}\text { deposited } \\
\left(\times 10^{19}\right)\end{array}$ & dep. efficiency \\
\hline Ex $_{\mathrm{fwd}}$ & $\mathrm{UV}$ & 11.9 & 16 & 1.2 & 1.0 & 2.8 & 11 & $40 \%$ \\
$\mathrm{Ex}_{\mathrm{fwd}}$ & $\mathrm{LV}$ & 4.9 & 23 & 5 & 2.0 & 5.5 & 18 & $33 \%$ \\
$\mathrm{Ex}_{\mathrm{rev}}$ & $\mathrm{UV}$ & 11.2 & 7.6 & 5 & 1.0 & 2.9 & 7.3 & $26 \%$ \\
$\mathrm{Ex}_{\mathrm{rev}}$ & $\mathrm{LV}$ & 4.2 & 7.6 & 25 & 2.3 & 6.6 & 10 & $15 \%$ \\
$\mathrm{Ex}_{\mathrm{src}}$ & $\mathrm{UV}$ & 9 & 20 & 1 & 0.56 & 0.6 & 0.84 & $14 \%$ \\
\hline
\end{tabular}

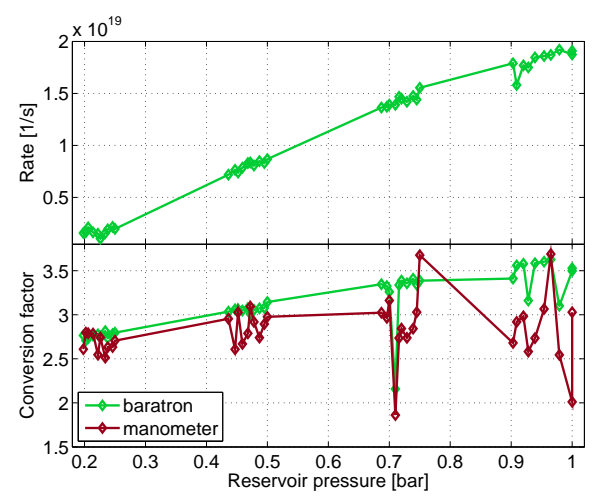

Figure 2. Calculated injection rates from pressure measurements with the baratron installed in the methane reservoir (top) [4]. Conversion factors for obtaining the actual injection rates from the baratron measurements, as well as from the additional manometer measurements typically carried out during the gas injection (bottom).

by using newly calibrated data for the injection rates and spectroscopic observations of the hydrocarbon puff. The effect of the methane source on the local plasma conditions is discussed using results from a linear gas injection ramp-up experiment. A crosscomparison of the spectroscopic and surface analysis data is made for the whole experimental series. A detailed interpretation of the results is left for section 3 .

\subsection{In-situ observations of the ${ }^{13} \mathrm{CH}_{4}$ puff}

The injection of ${ }^{13} \mathrm{CH}_{4}$ from the two divertor valves in AUG has been monitored in the past by using pressure measurements inside the gas reservoir. Recently, large discrepancies were found when comparing these rates with the in-vessel pressure measurements in AUG [5]. More dedicated recalibration efforts, by means of a two chamber experiment, have shown that the measurements inside the gas chamber underestimate the actual injection rate by a factor of $\sim 3$, see figure 2 . The discrepancy appears to have a weak dependence on the pressure inside the reservoir, but no variations between different gases $\left(\mathrm{CH}_{4}\right.$ and $\left.\mathrm{He}\right)$ has been observed. This implies that the quoted injection rates in earlier publications of local ${ }^{13} \mathrm{CH}_{4}$ injection in AUG $[4,8,9]$ 


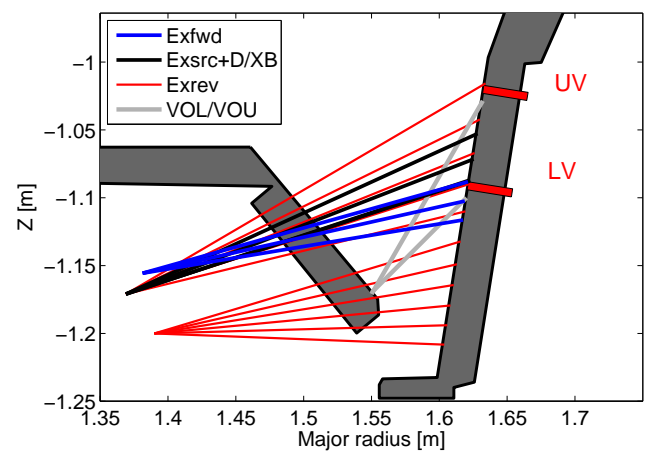

Figure 3. Spectroscopic lines-of-sight in the different campaigns. Similar setup was used in all campaigns for the larger spectroscopic cones looking at the two injection locations, labelled VOU/VOL for UV/LV respectively.

have been significantly underestimated. It is unclear whether the calibration of the gas-reservoir measurements has changed over the years but, starting from the first benchmarking of the pressure measurement system in 2007, conversion factors consistent with figure 2 have been observed.

The gas injection at the two exit holes was monitored in the experiments by measuring the spectroscopic emission from the methane puff. The primary signals measured were the emission from the so-called standard CD band at 429.5-431 nm [10] and the CII line at $426.7 \mathrm{~nm}$. These signals were used to identify the starting time of the injection and to compare the transport between hydrocarbons and carbon ions. Both measurements include contributions from the dissociation products directly from the injected methane $\left({ }^{13} \mathrm{CH}_{4}\right)$ and from the reflected or re-eroded hydrocarbons $\left({ }^{13} \mathrm{C}_{X_{1}}{ }^{12} \mathrm{C}_{X_{2}} \mathrm{H}_{Y_{1}} \mathrm{D}_{Y_{2}}\right.$, see also [11]). The wavelength resolutions of the spectrometers $\left(0.010 \mathrm{~nm} /\right.$ pixel in $\mathrm{Ex}_{\mathrm{fwd}}$ and $0.013 \mathrm{~nm} /$ pixel in $\mathrm{Ex}_{\mathrm{src}}$ and $\left.\mathrm{Ex}_{\mathrm{rev}}\right)$ would have allowed distinguishing between the emissions from the $\mathrm{CD}$ and $\mathrm{CH}$ molecules [12]. However, as shown later in Section 3, a large fraction of the injected hydrocarbons are promptly reflected, so that $\mathrm{CH}$ emission alone is not representative of all injected methane. The emission was measured along the LOS shown in figure 3, viewing the toroidal position of the exit holes with spot sizes of $\sim 1 \mathrm{~cm}$ diameter. These spots are drawn along the target tiles later in figure 7 . In addition, the emission from each valve was measured along two LOS with a spot sizes of $5 \mathrm{~cm}(\mathrm{VOL})$ and $8 \mathrm{~cm}(\mathrm{VOU})$ diameter. In $\mathrm{Ex}_{\mathrm{rev}}$, these wider LOS were connected to a spectrometer with a higher wavelength resolution of $0.004 \mathrm{~nm} /$ pixel.

Figure 4 shows the time evolution of the measured CD and CII emission from the upper valve. Comparison of the measured emission with the baratron signal, which closely follows the applied opening voltage of the valve, reveals a time delay of 0.40.8 seconds before the injected gas reaches the exit hole in the torus. Due to this time delay, associated with the molecular flow in the connecting tube, not all of the injected gas reaches the plasma before the discharge ends (note in particular $\mathrm{Ex}_{\mathrm{src}}$ in figure 4). Therefore, the total injected numbers given in table 2 have been calculated taking this delay into account. After the molecules reach the exit hole, it takes typically another 1-2 seconds for the emission to reach a steady value.

Figure 5 shows a qualitative comparison of the poloidal CD and CII emission profiles in the three experiments $\mathrm{Ex}_{\mathrm{fwd}}, \mathrm{Ex}_{\mathrm{src}}$ and $\mathrm{Ex}_{\mathrm{rev}}$. The $\mathrm{CD}$ band emission 


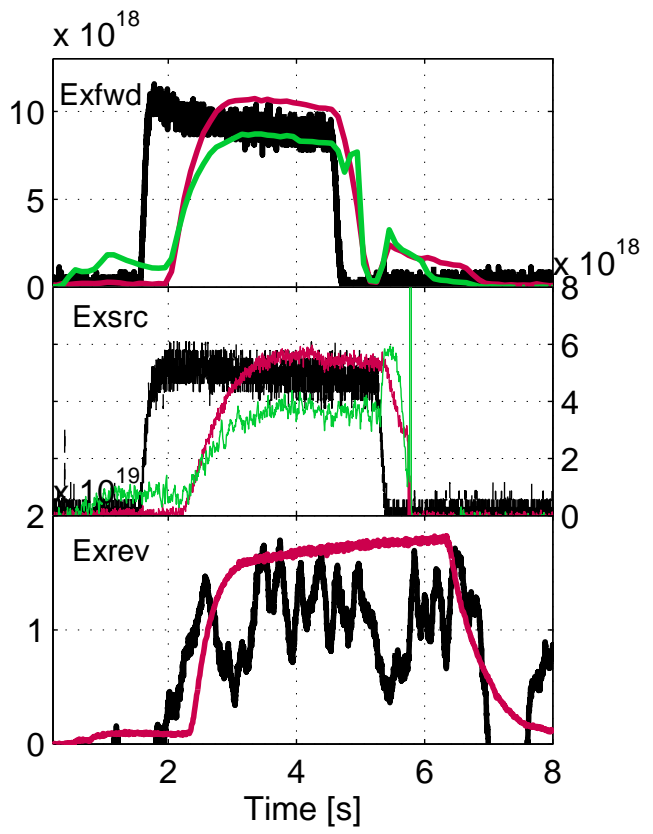

Figure 4. Injection rates derived from the UV baratron $\left(\mathrm{Ex}_{\mathrm{fwd}_{\mathrm{w}}}, \mathrm{Ex}_{\mathrm{src}}\right)$ and manometer $\left(\right.$ Ex $\left._{\mathrm{rev}}\right)$ measurements, including the conversion shown in figure 2 . Integrated emission from the CD band (red) and CII line at $426.7 \mathrm{~nm}$ (green) at the UV (VOU in figure 3) are shown in arbitrary units.

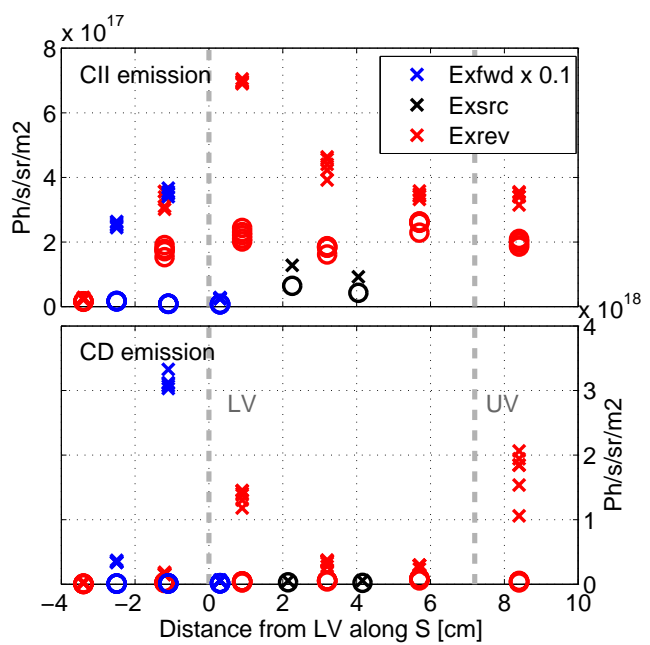

Figure 5. Integrated emission from the CD band and CII line along the Scoordinate in the three ${ }^{13} \mathrm{C}$ experiments, along the arrays drawn in Figure 3. The crosses represent the measured emission during the ${ }^{13} \mathrm{CH}_{4}$ puff and the circles give the measured values just before the injection begins (background emission). The active valves in each experiment are listed in table 2. Due to small differences in the toroidal positioning of the LOS, the absolute values are not directly comparable between the different campaigns. 


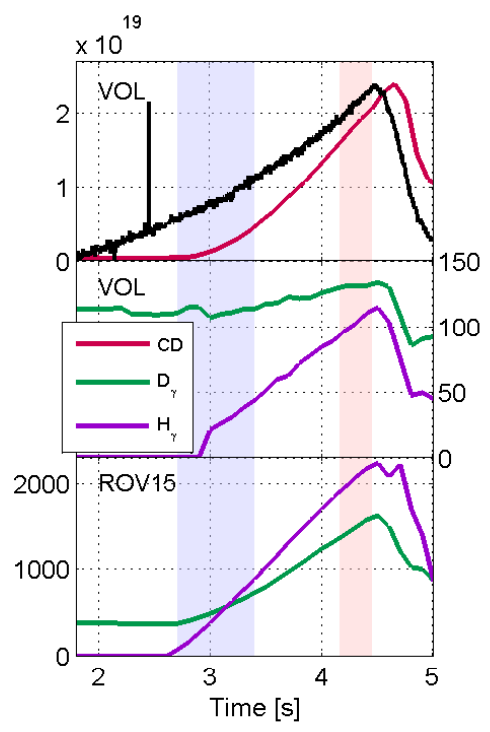

Figure 6. Time evolution of integrated emission from the CD band and the $\mathrm{D}_{\gamma}$ and $\mathrm{H}_{\gamma}$ lines during a linear ramp-up of the gas puff rate in \#23528. The measurements were carried out along two LOS, covering circular areas with $5 \mathrm{~cm}$ (VOL) and $1 \mathrm{~cm}$ (ROV15) diameters around the lower injection hole. The injection rate is shown in the top figure in black, as measured by a baratron system during a similar puff in \#23460. The estimated time ranges when the injection reaches the values used in $\mathrm{Ex}_{\mathrm{fwd}}$ and $\mathrm{Ex}_{\mathrm{src}}$ are highlighted with red and blue, respectively. The emission rates are given in arbitrary units.

is observed to be a lot more localized around the valves than the CII emission. This shows that the transport of hydrocarbons is limited around the source location, whereas further away the molecules have dissociated into pure carbon. In forward field, more emission is observed on the separatrix side of the exit holes, but the opposite observation is made in reversed field. This indicates that both hydrocarbons and carbon undergo transport poloidally along the target surfaces (as observed also for other impurities and SOL locations in Alcator C-mod [13]), and the direction of this transport depends on the field direction.

Spectroscopic measurements were used to study whether the gas injection rates were low enough not to perturb the divertor plasma conditions. In discharge \#23528, $\mathrm{CH}_{4}$ was injected from the two valves with linearly increasing puffing rate under similar divertor plasma conditions as in $\mathrm{Ex}_{\mathrm{fwd}}$. During the injection, the emission from the $\mathrm{CD}$ band and the neighbouring $\mathrm{D}_{\gamma}$ and $\mathrm{H}_{\gamma}$ lines at $434.1 \mathrm{~nm}$ were monitored with a wavelength resolution of $0.010 \mathrm{~nm} /$ pixel. In constant plasma conditions, and assuming negligible re-deposited carbon layer, the CD band emission should increase linearly with the hydrocarbon influx rate: $\phi=\frac{1}{[D / X B]} \cdot \Gamma_{\mathrm{CH}_{x}, \mathrm{CD}_{\mathrm{y}}}$, where $\phi$ is the measured photon flux from the $\mathrm{CD}$ band, and the $\mathrm{D} / \mathrm{XB}$-value describes the rate of dissociations with respect to excitations to the measured transition levels [11]. Figure 4 shows that a delay in the CD band emission is observed, similarly to the other experiments, after which the emission increases faster than the injection rate. This deviation could be associated with the initial ramp-up time of the gas injection rate, discussed earlier, decreasing the measured injection/emission delay time towards 
the end of the discharge. Another possibility is a growing contribution of re-eroded hydrocarbons as the carbon layer builds up. Therefore, no definite sign of perturbation can be identified in this comparison.

The effect of the methane puff on the background plasma was further analysed by comparing the $\mathrm{H}_{\gamma}$ and $\mathrm{D}_{\gamma}$ emission. The former results from hydrogen atoms dissociated from the injected methane molecules or released at the surface and the latter is typically associated with the recycling of the main plasma ions. Figure 6 shows the time evolution of the $\mathrm{D}_{\gamma}$ and $\mathrm{H}_{\gamma}$ lines along the wider $5 \mathrm{~cm}$ diameter LOS and a more localized $1 \mathrm{~cm}$ diameter LOS that both look at the LV during the ramp-up of the methane injection rate. At the location of the exit hole, $\mathrm{D}_{\gamma}$ emission starts to increase already at the very lowest injection rates that lead to $\mathrm{H}_{\gamma}$ emission. Assuming that the change in $\mathrm{D}_{\gamma}$ reflects the changes in plasma conditions experienced by the recycling neutrals, this implies that some perturbation is inherently involved in this experimental arrangement that involves injection through a hole. However, the possible perturbation appears to be very localized around the exit hole, as only a small change in $\mathrm{D}_{\gamma}$ is seen by the wider LOS.

\subsection{Results from post-mortem surface analyses}

After each experiment, the divertor tiles surrounding the injection holes were promptly removed from the torus for post-mortem analysis of the locally deposited ${ }^{13} \mathrm{C}$. The analysis was carried out for the three tiles surrounding the injection locations, see figure 7. When mounted on the divertor, the two leftmost tiles have a $5 \mathrm{~mm}$ wide gap between them. Due to tilting of the tiles, there is a small radial separation, the tile edge on the left protruding $0.8 \mathrm{~mm}$ deeper into the plasma than the adjacent tile edge on the right. The magnetic field makes about 1.5 degree incidence angle onto the tile surfaces such that the downstream direction is to the right in figure 7 . Therefore, the first $\sim 2 \mathrm{~cm}$ from the left edge of the injection tile is partially shadowed, with an average thickness corresponding to the plasma ion gyroradius, $r_{\mathrm{L}} \sim 0.3 \mathrm{~mm}$. As will be discussed in section 3, this partial shadow is likely to influence the impurity transport in this very thin volume close to the injection. Furthermore, flaking of the thick ${ }^{13} \mathrm{C}$ deposit was observed in this region during the removal of the tile, yielding an uncertainty of the local ${ }^{13} \mathrm{C}$ content.

Most of the measurements were carried out using the nuclear reaction analysis technique, NRA. The analyses were performed using $2.5 \mathrm{MeV}^{3} \mathrm{He}^{+}$ions and detecting protons originating from the reaction ${ }^{13} \mathrm{C}\left({ }^{3} \mathrm{He}, \mathrm{p}\right){ }^{15} \mathrm{~N}$ at $150^{\circ}$. This allows to determine the lateral distribution of ${ }^{13} \mathrm{C}$ within a $20 \%$ error [4]. In addition, parts of the deposition layers in $\mathrm{Ex}_{\mathrm{rev}}$ were analysed by secondary ion mass spectrometry, SIMS [14]. The SIMS analyses were made using a $5 \mathrm{keV} \mathrm{O}{ }_{2}^{+}$primary ion beam with a current of $500 \mathrm{nA}$ and a high mass resolution of $m / \Delta m \approx 2000$ to distinguish ${ }^{13} \mathrm{C}$ from closely lying isobars. The SIMS measurements concentrated in regions where in NRA the ${ }^{13} \mathrm{C}$ peak was overlapped by a much stronger D peak; no such limitation exists in SIMS.

Comparison of measured and injected ${ }^{13} \mathrm{C}$ shows that the highest deposition efficiencies are observed in $\mathrm{Ex}_{\mathrm{fwd}}$, with deposition tails formed in both toroidal directions. In this experiment, $40 \%$ of the deposition is found upstream (i.e. left) from the exit hole and the deposition efficiency is lower for the LV compared to UV. The tails have a downward deviation, towards the strike point, compared to the magnetic field direction. Right below the injection locations, in the partially shadowed area, 


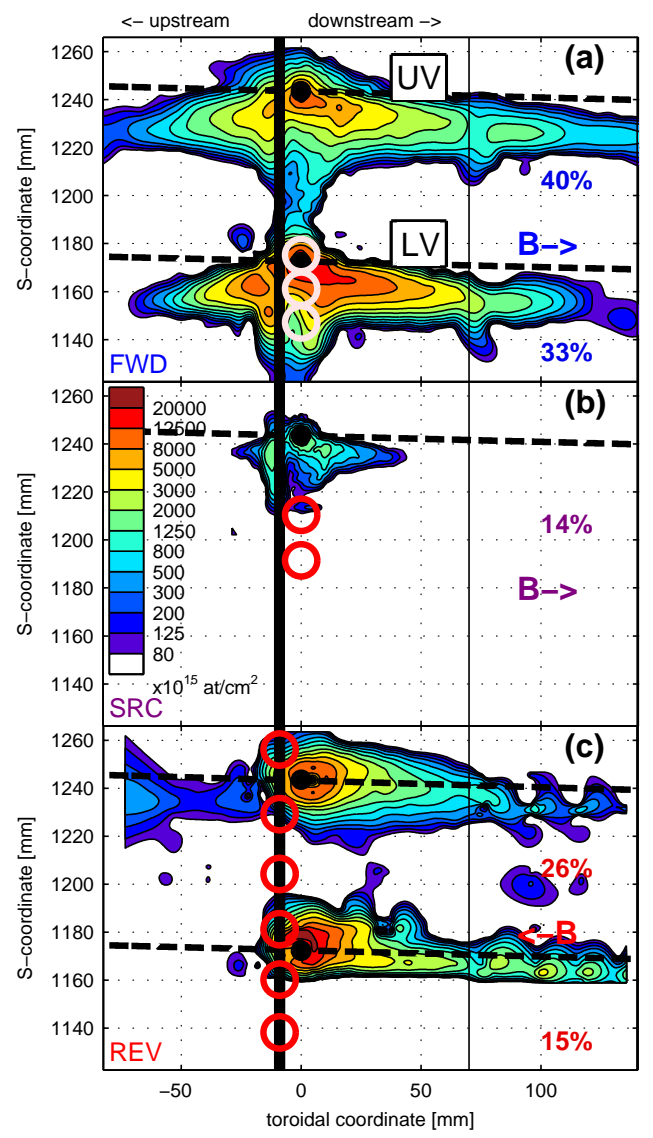

Figure 7. Measured ${ }^{13} \mathrm{C}$ deposition patterns in experiments $\mathrm{Ex}_{\mathrm{fwd}}$ (a), $\mathrm{Ex}_{\mathrm{src}}$ (b) and $\mathrm{Ex}_{\mathrm{rev}}$ (c). Figure (a) is reproduced from [4], with minor modifications. Figure (c) shows both the NRA data published in [5] and the new data from SIMS measurements. The blue circles indicate the spot sizes of the poloidally distrubuted LOS shown in Figure 3. The black vertical lines indicate the positions of the tile gaps, where measurements were not carried out.

there are deviations from the general pattern, such that the deposition extends further downwards and is suppressed close to the tile gap.

A significant reduction (by 70\%) in the re-deposition efficiency is observed in $\mathrm{Ex}_{\mathrm{src}}$ compared to $\mathrm{Ex}_{\mathrm{fwd}}$. The pattern, however, resembles those in $\mathrm{Ex}_{\mathrm{fwd}}$ : the downward deviation of the tail outside the shadowed area is similar to the one observed with the higher source rate. This supports the view that the possible perturbation caused by the puff is highly localized around the exit hole, where also the measurements have an uncertainty because of flaking of the deposit. The injection rate appears not to affect the transport of impurities further away from the exit hole, but it influences the balance between deposition and erosion rates. What is particularly visible with the lower source rate is the strong deposition on the protruding right edge of the upstream tile. Equal amounts of ${ }^{13} \mathrm{C}$ are observed upstream and downstream from the exit hole. The tails have a shorter extension in $\mathrm{Ex}_{\mathrm{src}}$ compared to $\mathrm{Ex}_{\mathrm{fwd}}$.

In reversed field configuration $\left(\mathrm{Ex}_{\mathrm{rev}}\right)$, the deposition efficiencies are reduced by 
$30 \%$ (UV) to $50 \%$ (LV) compared to Ex $\mathrm{x}_{\mathrm{fwd}}$. Only $20 \%$ of the deposited ${ }^{13} \mathrm{C}$ is found upstream of the injection. Unlike in $\mathrm{Ex}_{\mathrm{fwd}}$, the tails mainly follow the magnetic field lines, with a small upward deviation close to the exit holes. The patterns are generally more circular and localized in reversed field.

To summarize, re-deposition efficiencies in between $14-40 \%$ are measured within the three experiments. These are similar to the observations made at JET [15] whereas significantly lower local re-deposition with values between $0.2-9 \%$ has been measured on TEXTOR limiters [16]. This apparent geometry effect is discussed further in section 3 .

It should be noted that the surface roughness of the divertor tiles varied between the experiments. In the first two forward field experiments, the average roughness, $R_{\mathrm{a}}$, was $30-50 \mu \mathrm{m}$. In $\mathrm{Ex}_{\mathrm{rev}}$, the roughness was reduced to $R_{\mathrm{a}} \sim 1 \mu \mathrm{m}$. In the limiter experiments in TEXTOR, surface roughness has been observed to affect the

${ }^{13} \mathrm{C}$ deposition efficiency, by increasing it on rough graphite surfaces even by a factor of 5 compared to polished surfaces [16]. This was suggested to be due to impurities being trapped in between the roughness peaks. Unfortunately, we cannot apply these observations directly to the present analysis, as none of the tiles in the AUG experiments were polished, and no previous studies of ${ }^{13} \mathrm{C}$ deposition on tungsten surfaces with various $R_{\mathrm{a}}$ exist, particularly not for a divertor geometry.

A colorimetry analysis, based on photographs taken from the ${ }^{13} \mathrm{C}$ layer, was performed in [4] to investigate the effect of the very rough surfaces in $\mathrm{Ex}_{\mathrm{fwd}_{\mathrm{w}}}$. This analysis revealed large asymmetries in the ${ }^{13} \mathrm{C}$ deposition on the different sides of the surface roughness, with most deposition found on the surface parts facing the injection location. In contrary to [16], this suggests a low re-erosion/reflection of impinging carbon. Similar results were obtained for $\mathrm{Ex}_{\mathrm{rev}}$. Therefore, we cannot draw conclusions similar to [16] of the surface roughness effects, but have to leave this as an uncertainty in the interpretation until further experimental data is available.

\section{Interpretive ERO simulations}

To understand how the magnetic field direction and the local plasma conditions affect the migration of carbon, a detailed modelling of the ${ }^{13} \mathrm{CH}_{4}$ puffs in $\mathrm{Ex}_{\mathrm{fwd}}, \mathrm{Ex}_{\mathrm{src}}$ and $\mathrm{Ex}_{\mathrm{rev}}$ was performed. For this purpose, we used the ERO code [6], in combination with the plasma background models from SOLPS5.0, as derived in Part I. ERO is a Monte Carlo code that follows the kinetic 3D trajectories of various impurities which are injected or released to the plasma from a given material surface. It calculates both erosion and re-deposition of these impurities on a finite surface area. Impurity deposition patterns are therefore routinely obtained in the simulations, which makes the code ideal for interpreting the local ${ }^{13} \mathrm{C}$ deposition measurements.

In the following, the simulation method using ERO with SOLPS5.0 plasma backgrounds is first described. ERO is then used to model the ${ }^{13} \mathrm{CH}_{4}$ injection experiments, in comparison with the experimental data presented in section 2 . The differences in the migration of hydrocarbons and carbon, as well as neutrals and ions, are identified and the contribution of each species on the deposition pattern is shown. The migration mechanisms, including transport in the plasma and re-erosion/reflection at the surface, are analysed. Finally, conclusions on the effects of the magnetic field direction and local plasma conditions are drawn. 


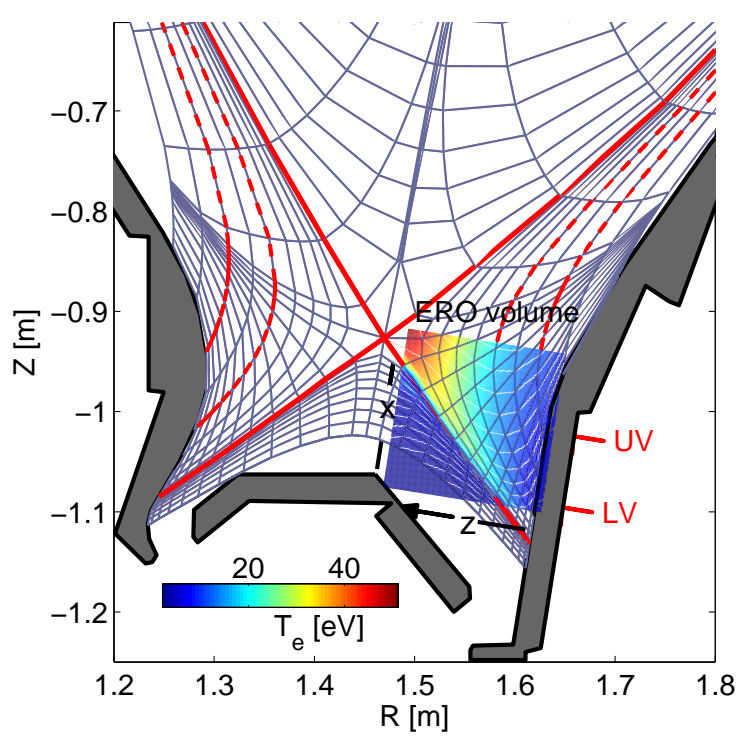

Figure 8. 2D cross-section of the ERO simulation box for the upper valve. The figure shows the electron temperature distribution in $\mathrm{Ex}_{\mathrm{rev}}$ after interpolation from the SOLPS grid (partially shown).

\subsection{Simulation setup with integrated SOLPS5.0 solutions}

In order to perform the necessary parameter studies, the ERO simulation volumes were limited to cover only the plasma regions relevant for local re-deposition of ${ }^{13} \mathrm{C}$. According to figure 7 , there is no overlap between the ${ }^{13} \mathrm{C}$ re-deposition patterns from the two sources and, therefore, the simulations could be carried out separately for each source. Figure 8 shows the poloidal cross-section of the ERO simulation volume for the UV, with respect to the SOLPS5.0 simulation grid in $\mathrm{Ex}_{\mathrm{rev}}$. In this $2 \mathrm{D}$ view, most of the divertor plasma is covered by extending the ERO grid $16 \mathrm{~cm}$ in both the poloidal ( $x$, along the target) and radial ( $z$, into the plasma) directions. In the toroidal direction ( $y$, along the target), the volumes extend $10 \mathrm{~cm}$ upstream and $30 \mathrm{~cm}$ downstream from the injection. A smooth and flat tungsten surface is specified for all the simulations, and the mixing of carbon and tungsten is taken into account by calculating the material concentrations in a specified interaction layer and iterating the surface evolution steps until a steady state is reached (homogeneous mixing model, HMM [17]). For calculating the erosion sources, the surface is divided into $5 \mathrm{~mm} \times 5 \mathrm{~mm}$ surface cells. The results are not observed to change significantly when increasing the resolution of the surface grid. The non-point ${ }^{13} \mathrm{CH}_{4}$ sources from the gas injection holes are represented by multiple point sources with cosine angular distribution and thermal energy distribution within a circle of $5 \mathrm{~mm}$ diameter.

ERO follows the trajectories of the test particles from both the ${ }^{13} \mathrm{CH}_{4}$ source and from the erosion sources. Ionized particles interact with the plasma background through Coulomb collisions, and dissociation/ionization reactions, including the full methane break-up chain, are treated probabilistically according to rate coefficients taken from atomic/molecular databases [18]. These processes depend on the plasma 
Table 3. Most frequent first reactions for $\mathrm{CH}_{4}$ molecules injected through the UV in experiment $\mathrm{Ex}_{\mathrm{fwd}}$. Other reactions have probabilities below $5 \%$ per reaction.

\begin{tabular}{lc}
\hline reaction & frequency \\
\hline $\mathrm{e}+\mathrm{CH}_{4} \rightarrow \mathrm{CH}_{3}+\mathrm{H}+\mathrm{e}$ & $29 \%$ \\
$\mathrm{e}+\mathrm{CH}_{4} \rightarrow \mathrm{CH}_{4}^{+}+2 \mathrm{e}$ & $26 \%$ \\
$\mathrm{e}+\mathrm{CH}_{4} \rightarrow \mathrm{CH}_{3}^{+}+\mathrm{H}+2 \mathrm{e}$ & $17 \%$ \\
$\mathrm{p}+\mathrm{CH}_{4} \rightarrow \mathrm{CH}_{4}^{+}+\mathrm{H}$ & $11 \%$ \\
\hline
\end{tabular}

density $n_{\mathrm{e}}$ and temperatures $T_{\mathrm{e}}$ and $T_{\mathrm{i}}$. To determine the frictional drag caused by the plasma, information on $v_{\|}$is needed in addition. Ions gyrate around the magnetic field lines and may experience drifts in the presence of an electric field or spatially varying magnetic field and, thus, knowledge of the plasma potential, $V_{\mathrm{p}}$, and $\mathbf{B}$ are required. These drifts are included in the modelled particle trajectories, which are based on full gyro-orbit calculation according to the Lorentz force. No cross-field diffusion was assumed for the modelled tracer molecules, as it is unclear how the global assumptions of anomalous diffusion (see figure 4 in Part I) should be applied to the various, possibly unthermalized hydrocarbons migrating in the vicinity of the target. For most of the modelled volume, all the aforementioned parameters can be extracted from the SOLPS5.0 solutions presented in Part I by interpolating them to the rectangular ERO grid. An example of the background $T_{\mathrm{e}}$ distribution is shown in figure 8 .

As the SOLPS5.0 solutions extend only to the edge of the magnetic presheath, the parameters in the remaining thin layer before the target must be calculated separately. Apart from $V_{\mathrm{p}}$, the plasma parameters in this region are assumed to remain constant in the $z$ direction. Due to the large angle of incidence of the magnetic field in the AUG divertor, most of the sheath potential drop occurs within the MPS. The MPS potential is calculated in ERO according to a simplified analytic formula: $V_{\mathrm{mps}}(\mathrm{d} z)=f \cdot V_{\mathrm{sh}} \mathrm{e}^{-\mathrm{d} z / r_{\mathrm{L}}}[6]$, where $\mathrm{d} z$ is the distance from the surface, $f \sim 0.9$ for the incidence angles in AUG and $V_{\mathrm{sh}} \sim-3.8 T_{\mathrm{e}} / e$ is the total potential drop within the sheath [19]. Unlike the electrostatic sheath, which has a thickness of only a few Debye lengths $\left(\lambda_{\mathrm{D}} \sim 0.01 \mathrm{~mm}\right)$, the MPS extends up to $l_{\mathrm{mps}} \sim 1 \mathrm{~mm}$.

In addition to the plasma parameters, the SOLPS5.0 solutions were used to determine the background impurity concentration. ERO takes into account the physical sputtering caused by the background plasma, background impurities, trace impurities and impurities eroded from the substrate. A constant carbon impurity percentage of $n_{\mathrm{e}}$ with an average charge 2+ was specified for each ERO simulation, according to the concentrations of $\mathrm{C}^{1-6+}$ in the SOLPS5.0 solutions at the injection locations. The chemical erosion yield of the re-deposited carbon layer, consisting of both background and injected carbon, was assumed to be $2 \%$ of the incoming $\mathrm{D}^{+}$ flux. This yield could be varied independent of the assumptions for $\mathrm{Y}_{\text {chem }}$ used in the SOLPS5.0 simulations, in which full carbon wall elements were specified.

\subsection{Effect of hydrocarbons and neutrals on the migration of carbon}

The migration length scale and local deposition efficiency are largely determined by the penetration depth of the injected impurities. When a neutral is born as a result of dissociation, it may return to the surface provided that its velocity is towards the 


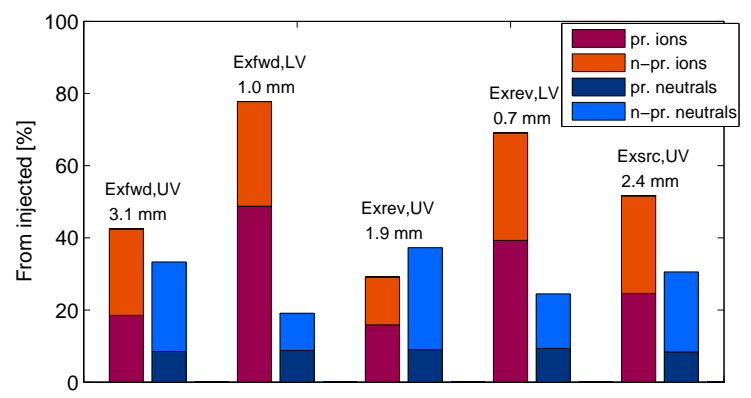

Figure 9. Fractions of returning particles that undergo only 1 reaction (prompt) or several (non-prompt) during their plasma dwell time. The average ionization depths of all the injected particles are given on top of the bars.

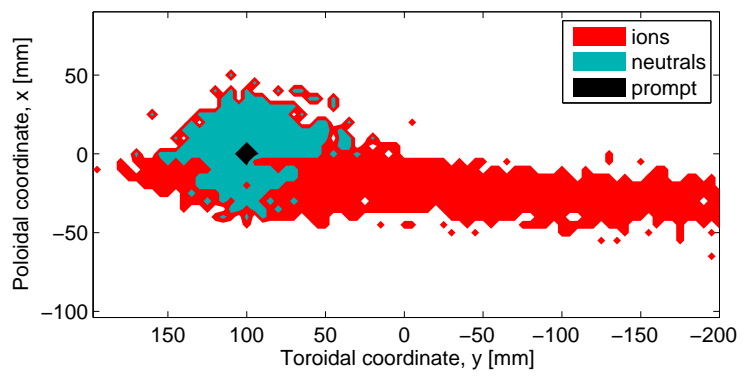

Figure 10. Distribution of the first incidences for the injected particles returning to the surface. The colours are determined by the majority species returning to the cell. Only cells that receive at least $0.1 \%$ of the injected particles are coloured.

target and the ionization mean-free path, $l_{\mathrm{iz}}$, is sufficiently long compared to $\mathrm{d} z$. The re-deposition of ions is more complicated, because the ions are affected by the plasma and by the strong electric fields close to the target. If the molecules ionize within the MPS, they may be guided promptly back to the surface by the strong electric field, $\mathbf{E}_{\mathrm{mps}}=-\nabla V_{\mathrm{mps}}$. Prompt re-deposition is particularly associated with the heavy hydrocarbons in the beginning of the dissociation chain. According to table 3 , the first dissociation/ionization reactions typically yield hydrogen-rich ions and atoms; $85 \%$ of the product molecules have at least three $\mathrm{H}$ atoms, whereas the yield of pure carbon is less than $1.5 \%$.

Figure 9 shows the distribution of ions and neutrals returning to the modelled surface area from the injection. In all cases, nearly half of the returning particles are promptly returning hydrocarbons. These 'prompt' particles are defined here as particles that undergo only one reaction before impinging on the target; the rest are called 'non-prompt'. In each field direction, the reduction of ionization length is found to lead to more particles impinging on the surface. However, the fractions of impinging particles are smaller in reversed compared to forward field. Figure 10 shows that the promptly returning ions and neutrals typically impinge on a circular area of $1 \mathrm{~cm}$ in diameter around the exit hole.

The non-prompt neutrals return to the surface forming a circular pattern around the exit hole, but with a larger extension compared to prompt particles. Due to the 


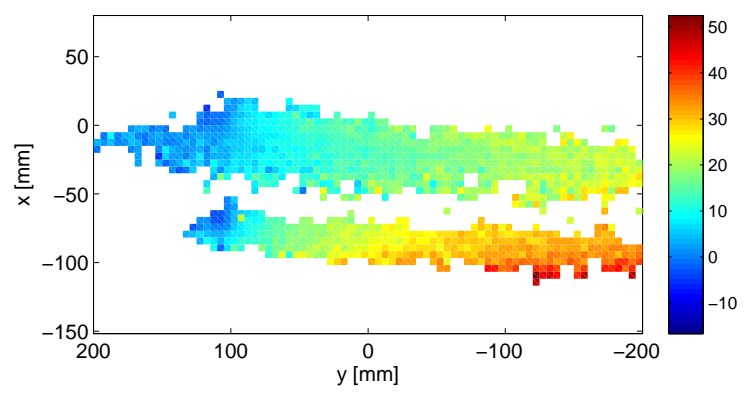

Figure 11. Angles of incidence of returning ions in $\mathrm{Ex}_{\mathrm{fwd}}$, calculated in the $y-z$ plane. The angles $90^{\circ}$ and $-90^{\circ}$ correspond to the negative and positive $y$ directions, respectively.

small angle between the magnetic field and the surface, the non-prompt ions that travel outside the MPS may become transported over large toroidal distances before impinging on the target. Because of the fast dissociation of hydrocarbons, most of the non-prompt ion deposition is due to pure carbon in various ionization states. According to figure 11, the parallel velocity of ions increases when travelling away from the injection, causing the impact angles to approach the impact angle of the magnetic field. This could explain the asymmetric deposition observed in colorimetry, recall section 2.2 .

The observations show that the re-deposition of ${ }^{13} \mathrm{C}$ is influenced by transport in two qualitatively different regions: the MPS and the plasma region outside the MPS. The majority of the pattern is caused by particles travelling outside the MPS, whereas prompt re-deposition can significantly contribute to the layer thickness close to the exit hole. As noted in section 2, the latter is a region where the protruding tile edge and impurity puff can influence the plasma conditions and where the measured ${ }^{13} \mathrm{C}$ content is uncertain because of flaking of the deposit. Furthermore, the absence of a material surface at the exit hole could influence the electric field in the MPS, making the code calculations uncertain. Therefore, our models should be most applicable for the regions outside the MPS, and thus for interpreting the patterns as a whole. Since the particles travelling outside the MPS are mainly carbon ions, these patterns characterize the migration of impurities in general.

\subsection{Effect of local plasma conditions on the re-deposition efficiency}

The deposition pattern and deposition efficiency are further determined by the reflection and re-erosion of the impinging impurities. Reflection of carbon atoms and ions is calculated in ERO using results from the TRIM code but data for the reflection of hydrocarbons is not yet thoroughly implemented in the divertor version. Often an effective reflection probability, $R_{\text {eff }}$, is used in the simulations. Molecular dynamics simulations indicate, however, that the reflection probability of hydrocarbon molecules decreases with increasing impact energy, $E_{\mathrm{imp}}$, and decreasing hydrogen content $[20,21]$. In this paper, we wished to test this type of species-dependent reflection model, assuming for simplicity an a-CH layer and using an average impact energy for each molecule.

Figure 12 shows the impact energies of the impinging trace impurities for two 


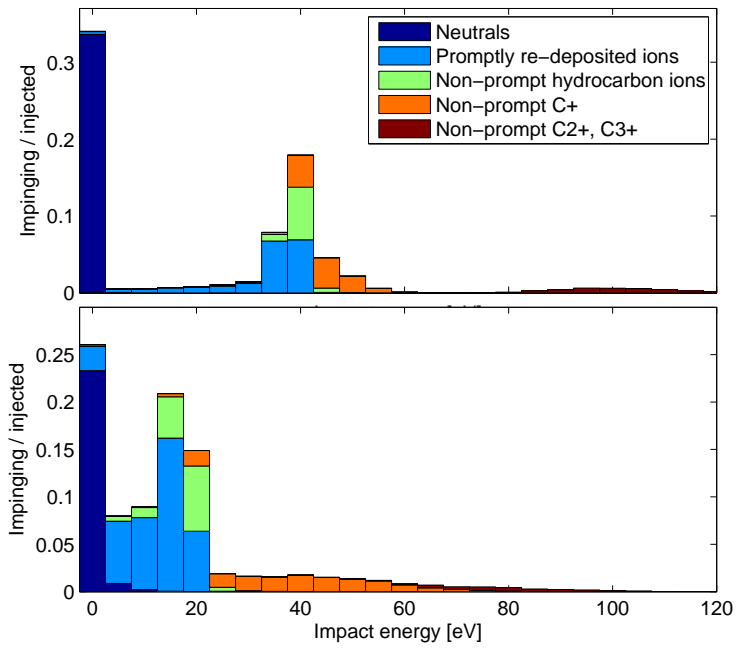

Figure 12. Impact energies of returning particles at the UV in $\mathrm{Ex}_{\mathrm{fwd}}$ (top) and at the LV in Exrev (bottom).

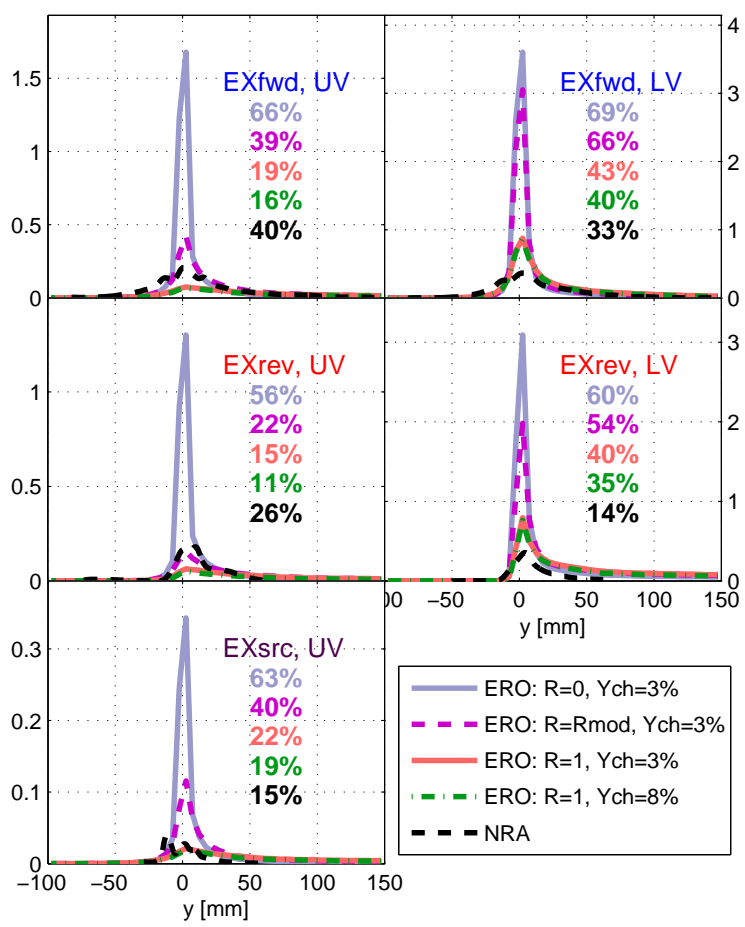

Figure 13. Toroidal distributions of the modelled and measured ${ }^{13} \mathrm{C}$ deposition $\left(\left[1 \times 10^{20} \mathrm{~cm}^{-1}\right]\right)$, integrated over the poloidal direction. The four different reflection models are described in the body text. Local deposition efficiencies in each case are marked on the figures. The dashed black lines show the results from surface analysis. 


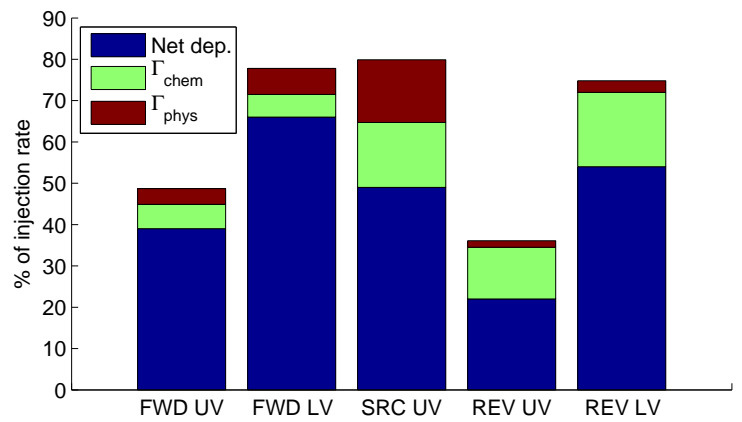

Figure 14. Fractions of deposited and re-eroded particles during one time step when a steady state is reached in the ERO simulations. The numbers are scaled with the injected number of particles per time step.

cases with different local plasma conditions: injections from the UV in $\mathrm{Ex}_{\mathrm{fwd}}$ and from the LV in $\mathrm{Ex}_{\mathrm{rev}}$. Due to the low collisionality in $\mathrm{Ex}_{\mathrm{fwd}}$, the impurities are not thermalised during their short dwell time in the plasma. The neutrals arrive with very low energies and, therefore, have a high reflection probability: $R_{\mathrm{n}}=0.65-1$. The ions, however, are accelerated in the sheath and arrive with high energies and have low average reflection probabilities: $R_{\mathrm{i}}=0.1-0.5$. For the low $T_{\mathrm{e}}$ and high $n_{\mathrm{e}}$ in $\mathrm{Ex}_{\mathrm{rev}}$, a bigger scatter in $E_{\mathrm{imp}}$ is observed due to higher collisionality and smaller $V_{\mathrm{sh}}$. The reflection of $\mathrm{CH}_{3}^{+}$and $\mathrm{CH}_{4}^{+}$increases to almost $R_{\mathrm{i}}=1$, whereas for the other hydrocarbons this effect is smaller and they can have a significant sticking.

In addition to the reflection properties, the net amount of re-deposited ${ }^{13} \mathrm{C}$ is determined by the physical and chemical erosion of the carbon layer. In the present modelling, both $Y_{\text {chem }}$ and $Y_{\text {phys }}$ are multiplied by a factor of $~ 1.5$, thus assuming $\mathrm{Y}_{\text {chem }}=3 \%$, to account for the injection-free periods in the experiments that increase the average erosion of the layers compared to the injection sources, recall section 2.1. Furthermore, the extensive modelling carried out for TEXTOR limiter experiments shows that an enhanced in-situ re-erosion yield for re-deposited hydrocarbon layers, due to bombardment by both $\mathrm{D} / \mathrm{H}$ and hydrocarbons, is required to match the measured ${ }^{13} \mathrm{C}$ deposition patterns [22]. To show how the various assumptions on hydrocarbon sticking and re-erosion influence the deposition layer, we present the following variations. (i) All hydrocarbons stick to the surface and the chemical erosion yield is $3 \%$. (ii) The reflection coefficient is assigned separately for each hydrocarbon molecule and charge state according to the average impact energies recorded for these molecules, $R\left(\bar{E}_{\text {imp }}\right)$. An enhanced re-erosion is included in the values of $R$ by assuming that $80 \%$ of the deposited hydrocarbons are instantaneously re-eroded [22]: $R_{\text {mod }}=R_{\text {enh }}+\left(1-R_{\text {enh }}\right) R\left(\bar{E}_{\text {imp }}\right)$, with $R_{\text {enh }}=0.8$. (iii) All hydrocarbons are reflected, and $\mathrm{Y}_{\text {chem }}=3 \%$. (iv) All hydrocarbons are reflected, and an enhanced chemical re-erosion yield is assumed, $\mathrm{Y}_{\text {chem }}=8 \%$. The last assumption corresponds to a typical assumption made in TEXTOR [22] and JET [23] modelling.

Figure 13 shows the toroidal profiles of the steady-state ${ }^{13} \mathrm{C}$ deposition layers, integrated along the $x$-direction, for the various assumptions described above. One sees that with all these assumptions, the deposition efficiencies are an order of magnitude higher than in TEXTOR, where $0.2-9 \%$ local re-deposition efficiencies have been measured. This appears to be an effect of the geometry; a divertor geometry allows for a larger volume of ionization that is magnetically connected to the material surface 
than a test limiter geometry. However, a simultaneous agreement with the experiments using one reflection model for all five cases can not be reached. When the ionization length is the longest (UV, see also figure 9), the best match is obtained with the non-negligible sticking assumption that gives the highest reflection probability for the heavy hydrocarbons re-depositing close to the exit hole (case (ii)). When the ionization length is of the order of the magnetic presheath thickness (LV), the redepositing hydrocarbons yield a peak in the deposition close to the hole, and a better match to the experiments is obtained when all hydrocarbons are reflected.

As noted earlier, there are large uncertainties in both modelled and measured re-deposition of impurities in the region close to the exit hole. Therefore, the discrepancies described above are not surprising. However, in $\mathrm{Ex}_{\mathrm{rev}}$ the layer thickness is overestimated along the whole toroidal length around the LV injection, even when negligible sticking and enhanced re-erosion is assumed. Since this is the case with the shortest $l_{\mathrm{iz}}$, a large fraction of transport takes place in the MPS. Therefore, we suspect that in reality there must be deviations from the MPS models used in the present analysis. However, detailed investigations of conditions in this region would require 3D simulations of the MPS region, including the effects of the tile edges, which were not feasible during the present work. For consistency, the rest of the analysis uses the assumptions in case (ii), which on average yield the best agreement with the measurements.

Re-erosion is found to be dominated by the background deuterium, whereas the deposited background carbon impurities reduce the net erosion of the tracer by decreasing its concentration in the layer. Figure 14 shows the fractions of ${ }^{13} \mathrm{C}$ effectively re-deposited during an ERO iteration step, together with the fractions of physically and chemically re-eroded ${ }^{13} \mathrm{C}$. The numbers are scaled by the injection rates, and their sum gives the gross deposition. In reversed field, a larger flux of chemically eroded particles, $\Gamma_{\text {chem }}$, is obtained due to the higher deuterium fluxes compared to forward field. Again, a higher $\Gamma_{\text {phys }}$ is obtained in forward field because of a higher $T_{\mathrm{e}, \mathrm{ot}}^{\mathrm{pk}}$. Due to the small injection rate used in $\mathrm{Ex}_{\mathrm{src}}$, the re-erosion fluxes are large compared to the impinging ${ }^{13} \mathrm{C}$ fluxes but, because of the smaller $l_{\mathrm{iz}}$ and increased background carbon deposition, similar net deposition as at the UV in $\operatorname{Ex}_{\mathrm{fwd}_{\mathrm{w}}}$ is obtained in the modelling.

The modelled deposition efficiency for $\mathrm{Ex}_{\mathrm{src}}$ disagrees with the experiment, where a factor of 2 lower value is obtained. We suspect that this is related to $\mathrm{C} / \mathrm{W}$ mixing effects that become more pronounced when the formation of re-deposited layer is slow and the layer thickness is small. Such features have been observed when modelling TEXTOR experiments, in which the deposition efficiency of ${ }^{13} \mathrm{C}$ was found to be smaller on W compared to C, and covering a five times smaller area [24]. It was concluded that ERO-HMM could not reproduce these observations. They could only be modelled using a refined approach based on direct coupling of ERO and SDTrimSP codes, which allows to track the dynamics of depth-resolved concentrations of elements in the interaction layer. Therefore, it appears likely that coupled ERO-SDTrimSP simulations would reproduce the experimental observations in $\mathrm{Ex}_{\mathrm{src}}$ better than the ERO-HMM used here. As the present work emphasizes on the integration of plasmaimpurity modelling, we considered such detailed investigations on material mixing to be outside the scope of this paper. 


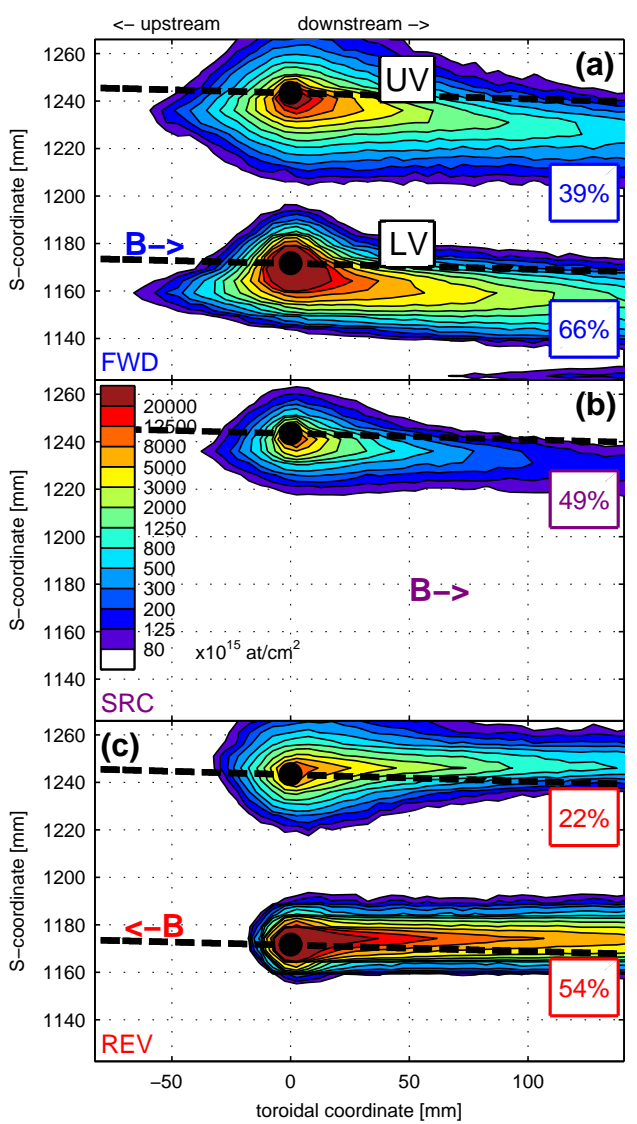

Figure 15. 2D deposition patterns modelled by ERO, using plasma backgrounds from the SOLPS simulations. The legends show the calculated re-deposition efficiencies.

\subsection{Effect of field reversal on ${ }^{13} \mathrm{C}$ migration}

We summarize the effects of field reversal on the local migration of ${ }^{13} \mathrm{C}$ by considering the modelled 2D deposition patterns shown in figure 15. As was shown earlier, the deposition patterns are largely determined by impurities that are not promptly redeposited from the MPS, but which undergo transport in the plasma background fully described by the SOLPS5.0 solutions. The re-depositing neutrals form a circular pattern around the exit hole, whereas the ions produce the long tails in the toroidal direction. Previous works have identified that the transport of ions is influenced by friction with the background plasma ions and an $\mathbf{E} \times \mathbf{B}$ drift perpendicular to the field lines $[5,9,25]$. These mechanisms largely determine the shape of the deposition tails, as is described in the following. It is worth noting that, this close to the target, thermal forces and $E_{\|}$have a negligible contribution in the parallel transport [25], and the deposition tails can be reproduced without assuming cross-field diffusion of impurities.

In forward field, the majority of the ${ }^{13} \mathrm{C}$ deposition is due to ions, as was shown in figure 9. Due to the low $n_{\mathrm{e}}$ and high $T_{\mathrm{e}}$, the collisionality of the plasma is low and 


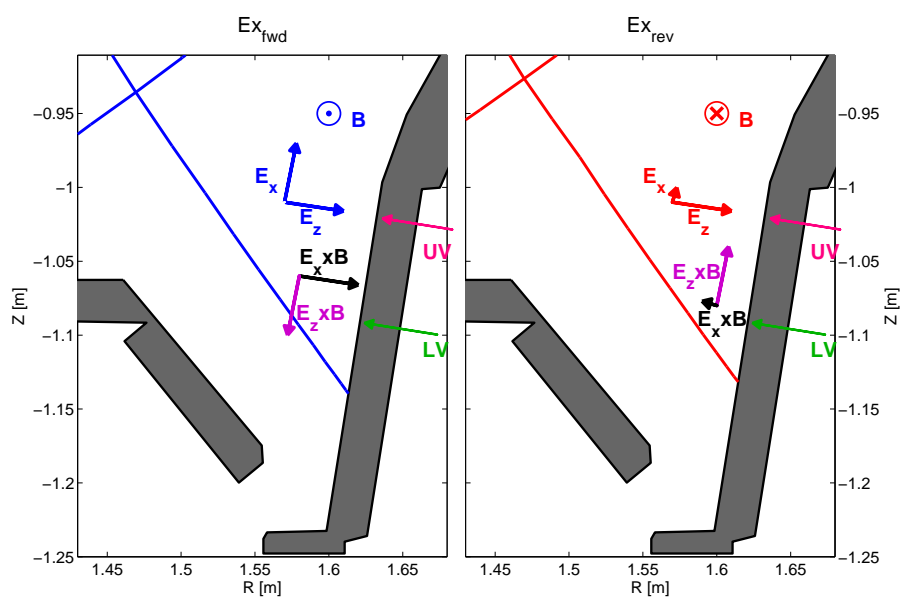

Figure 16. Illustration of the relative magnitudes and directions of the two electric field components perpendicular to the magnetic field, as calculated with SOLPS5.0 in Part I. The blue and red colours refer to the forward and reversed field plasmas, respectively.

the impurities experience small friction force. This allows them to travel upstream from the injection, against the plasma flow [9]. The ions may deposit on the upstream side of the exit hole if the $\mathbf{E} \times \mathbf{B}$ drift has a favourable direction and magnitude. As discussed in Part I, the electric field in the divertor in $\mathrm{Ex}_{\mathrm{fwd}}$ has two equally large components perpendicular to the magnetic field: in the direction towards the target $\left(E_{z}\right.$, parallel to $\left.\mathbf{E}_{\mathrm{mps}}\right)$, and parallel to the target $\left(E_{x}\right)$. Therefore, the $\mathbf{E} \times \mathbf{B}$ drift has components parallel to the surface, towards the strike point $\left(\mathbf{E}_{\mathbf{z}} \times \mathbf{B}\right)$, and towards the surface $\left(\mathbf{E}_{\mathbf{x}} \times \mathbf{B}\right)$, as visualized in figure 16. The first component causes the deviation of the deposition tails from the magnetic field lines. The second component increases the fraction of ions returning to the surface, particularly from regions outside the MPS. The deposition efficiencies can be increased up to a factor of 2 by the $\mathbf{E}_{\mathbf{x}} \times \mathbf{B}$, if only pure carbon is assumed to stick on the surface [25]. Furthermore, toroidally extended deposition tails can form upstream only as a result of this drift.

In reversed field, the higher $n_{\mathrm{e}}$ and lower $T_{\mathrm{e}}$ in the outer divertor result in more neutral dissociation products compared to forward field, recall figure 9 . The neutrals dominate the deposition close to the valves where a circular pattern is observed both in experiments and in modelling. Because of a high collisionality, the friction force is stronger compared to forward field and the ions are dragged into the downstream direction. The change in collisionality to a higher level has a stronger effect on the pattern than the reduction in parallel flow compared to forward field (see Part I). The direction of the electric field is slightly different from forward field, with a larger fraction of potential drop now in the direction towards the target (parallel to $\mathbf{E}_{\mathrm{mps}}$ ), see figure 16. The $\mathbf{E}_{\mathbf{z}} \times \mathbf{B}$ drift (as well as the $\mathbf{E}_{\mathrm{mps}} \times \mathbf{B}$ drift) produces the poloidal deviation of the deposition tail from the magnetic field lines, which in reversed field is away from the strike point. The modelled $\mathbf{E}_{\mathbf{x}} \times \mathbf{B}$ component, guiding the particles away from the surface in reversed field, is weaker than the corresponding component in forward field. The change in the $\mathbf{E}_{\mathbf{x}} \times \mathbf{B}$ drift with the field reversal could be seen in figure 14 to lead to smaller deposition efficiencies for all reflection models, despite the reduction of ionization length compared to forward field. 


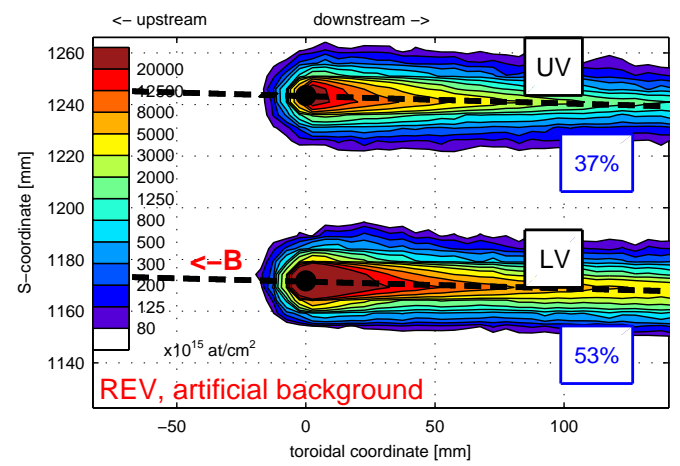

Figure 17. 2D deposition patterns in $E x_{\text {rev }}$, when an artificial plasma background that is fitted to the target Langmuir probe measurements (see Part I) is used in the simulations.

Both the observed effects of the $\mathbf{E} \times \mathbf{B}$ drift and the plasma collisionality can be argued to be important for global migration of impurities, as they define the direction where the impurities escape the divertor and at which rate they cross the PFR or outer SOL boundaries, see also $[26,27]$. Furthermore, they can influence material mixing in a multi-material target design, such as that proposed for ITER [3]. It is important to note that the drift effects are pronounced for eroded impurities that may have small parallel velocities before they are thermalized in collisions.

In the present modelling, there is no significant spatial variation in the patterns, due to small variations in the electric field properties and collisionality in the SOL. Particles in reversed field are primarily transported downstream in the toroidal direction, with $30 \%$ of the ${ }^{13} \mathrm{C}$ injected from the $\mathrm{LV}$ and $60 \%$ of those injected from the UV finally escaping the simulation volume through the toroidal boundary $30 \mathrm{~cm}$ downstream from the injection. The downstream direction dominates also in forward field with $20-50 \%$ escape fractions but, in addition, there is a significant transport poloidally towards the strike point. From the ${ }^{13} \mathrm{C}$ injected at the $\mathrm{LV}, 5 \%$ travels across the separatrix and is lost through the PFR boundary.

\subsection{Validity of the SOLPS5.0-ERO simulations}

Finally, we wish to analyse the validity of the modelled transport pathways when using SOLPS5.0 plasma background. In forward field, the SOLPS5.0 solutions agree well with the measurements in the outer divertor, and the main characteristics of the deposition patterns are also well reproduced in the modelling. We suspect that in the experiment the measured upstream deposition is enhanced due to the protruding tile on the left from the injection, causing more upstream deposition than what is observed in the simulations. The modelled transport towards the strike point agrees with the spectroscopic measurements presented in figure 5, which show that CII emission is seen only downwards from the injection. A slightly higher $E_{r}$, possibly suggested by the upstream measurements discussed in Part I, could result in a higher re-deposition efficiency. This, however, would likely remain within the uncertainty range of the measurements. Therefore, we conclude that the presented outer divertor impurity transport models appear realistic in forward field.

The discrepancies with measurements are larger in reversed field. At the LV, 
the modelled upward deviation of the tail is stronger compared to the experiment. It is unclear whether this is due to a wrong potential distribution in the SOLPS5.0 solution, or due to inaccurate description of transport and ionization within the MPS. For comparison, we show in figure 17 the deposition pattern in $\mathrm{Ex}_{\mathrm{rev}}$ when using an artificial background plasma that has constant plasma parameters along a given flux tube, fitted to the target Langmuir probe measurements. In these simulations, the divertor electric field consists of only the analytically calculated electrostatic sheath and magnetic presheath and, consequently, no poloidal deviation of the deposition tails from the magnetic field lines is observed. One sees that the modelled deposition patterns at the two valves are very similar to each other, in line with the flat target profiles measured by the probes. According to the NRA measurements, however, both the patterns and the deposition efficiencies at the UV and LV should differ from each other, and there should be poloidal deviations in the tails. It is apparent that the probe measurements alone do not give sufficient information about the outer divertor plasma in this experiment, for the purpose of impurity migration studies. Furthermore, since the SOLPS5.0 calculations disagree with the probes, it appears that the reversed field divertor plasma conditions are not yet thoroughly understood. We therefore conclude that more efforts have to be done to characterize the divertor conditions in reversed field experimentally and to investigate in detail the MPS properties and their effect on impurity deposition.

\section{Discussion and conclusions}

The paper presented results from ${ }^{13} \mathrm{CH}_{4}$ injection experiments in low-density Lmode discharges in ASDEX Upgrade, performed with both forward and reversed magnetic field. Part I showed that the field reversal leads to significant changes in the outer divertor plasma conditions. Here we showed that distinctly different local ${ }^{13} \mathrm{C}$ deposition patterns are also measured in the two opposite magnetic field directions. Detailed ERO simulations were performed to interpret the deposition patterns, using the SOLPS5.0 solutions derived in Part I for the outer divertor plasma. The changes in local migration were found to be due to both the changes in local plasma conditions and the reversal of the cross-field drifts of the impurities.

In forward field, the ${ }^{13} \mathrm{CH}_{4}$ injected from the vertical outer target was observed to form deposition tails in both toroidal directions, with a deviation from the magnetic field line towards the strike point. The pattern was found to result from low collisionality, allowing the ions to travel upstream from the injection, and from the $\mathbf{E} \times \mathbf{B}$ drift, transporting particles towards the strike point and back towards the target surface. In reversed field, the collisionality was higher and, therefore, no upstream transport was observed. Deviation from the magnetic field line was smaller compared to forward field and, because of reversed $\mathbf{E} \times \mathbf{B}$ drift, it was directed towards the outer SOL.

The injected ${ }^{13} \mathrm{CH}_{4}$ molecules were found to dissociate and ionize fast, with a significant fraction of ionizations occurring within the magnetic presheath. This raised an uncertainty in the modelling, which could not resolve the detailed effects of the injection hole, impurity puff and variations in tile alignment on the magnetic presheath. The strong decay of electric potential in this region was described by a commonly used exponential model, which was found to direct majority of the ionized molecules back to the surface. These returning hydrocarbons contributed to the thickness of the layer close to the injection, which was found to exceed the measured 
value unless an enhanced in-situ re-erosion of the hydrocarbons was assumed.

The method of injecting the methane gas through a $0.5 \mathrm{~cm}$ diameter hole was found to inherently lead to a local perturbation of the plasma in the near vicinity of the hole. Although this perturbation was localized enough not to have major influence on the patterns, an influence on the deposition of hydrocarbons and, thus, on the deposition efficiency appears likely. In addition to the mentioned uncertainties in magnetic presheath, this perturbation could explain why different reflection and re-erosion assumptions were required in each case to match the modelled deposition peaks and efficiencies with experimental results. Therefore, the often made assumption of enhanced re-erosion of hydrocarbons [22] could not be verified in these codeexperiment comparisons.

The local deposition efficiencies observed in a vertical divertor geometry were an order of magnitude higher than in TEXTOR limiter experiments. The simulations reproduced this large difference for a range of hydrocarbon sticking assumptions. Therefore, the difference was attributed to the differing surface geometries near the injection.

Those hydrocarbons that escaped the magnetic presheath dissociated into carbon atoms/ions, which could become transported over long distances or even escape the divertor plasma. The ${ }^{13} \mathrm{C}$ deposition patterns observed on the tiles were found to be largely determined by the carbon ions, and therefore characterized the transport of impurities in general. Outside the magnetic presheath, the plasma backgrounds were fully described by the SOLPS5.0 solutions. Therefore, the modelled main migration pathways were found to be sensitive to the validity of the SOLPS5.0 calculations.

Overall, it was shown that the SOLPS5.0-ERO simulations can reproduce well the measured deposition patterns, at least in cases when a match to the measured divertor conditions is obtained, the average ionization length is larger than the magnetic presheath thickness, and material mixing does not play an important role. The observed variations in the transport affect the rate and direction of impurity walking by subsequent re-erosion and re-deposition steps. Therefore, it was found that the divertor plasma conditions can have significant implications for both global migration and mixing of materials inside the divertor.

\section{Acknowledgments}

The authors would like to thank Mr. Gilbert de Saint-Aubin for his support in the NRA measurements of the ${ }^{13} \mathrm{C}$ re-deposition, and Prof. Ratko Janev and Dr. Sebastijan Brezinsek for useful discussions. This work, supported by the European Communities under the contracts of Association between EURATOM-Tekes, IPPEURATOM and EURATOM-FZJ, was carried out within the framework of the Task Forces on Plasma Wall Interactions and Integrated Tokamak Modelling of the European Fusion Development Agreement. The views and opinions expressed herein do not necessarily reflect those of the European Commission. The work was partially funded by the Academy of Finland project No. 134930.

\section{References}

[1] Kreter A et al, 2009 Journal of Nuclear Materials 390-391 38-43

[2] Stangeby P, 2011 J. Nucl. Mater. 415(1, Supplement) S278 - S283

[3] Pitts R A et al, 2009 Physica Scripta 2009(T138) 014001 
Outer divertor of ASDEX Upgrade in low-density L-mode discharges... - Part II 23

[4] Pugno R et al, 2009 J. Nucl. Mater. 390-391 68-71

[5] Aho-Mantila L et al, 2011 J. Nucl. Mater. 415(1, Supplement) S231 - S234

[6] Kirschner A et al, 2000 Nucl. Fusion 40989

[7] Ding R et al, 2011 Journal of Nuclear Materials 415(1, Supplement) S270 - S273

[8] Pugno R et al, 2005 J. Nucl. Mater. 337-339 985-9

[9] Aho-Mantila L et al, 2009 Phys. Scripta T 138. 014019

[10] Brezinsek S et al, 2004 Physica Scripta 2004(T111) 42

[11] Brezinsek S et al, 2007 J. Nucl. Mater. 363-365 1119-1128

[12] Brezinsek S et al, 2009 Physica Scripta T138 014022

[13] Jablonski D et al, 1997 Journal of Nuclear Materials 241243(0) $782-787$

[14] Hakola A et al, 2010 Plasma Phys. Control. Fusion 52(6) 065006

[15] Coad J et al, 2007 Journal of Nuclear Materials 363-365 287 - 293

[16] Kreter A et al, 2008 Plasma Physics and Controlled Fusion 50(9) 095008

[17] Kirschner A et al, 2009 J. Nucl. Mater. 390-391 152-155

[18] Janev R and Reiter D, 2002. Jül report Jül-3966

[19] Stangeby P, 2000 The Plasma Boundary of Magnetic Fusion Devices. IOP Publishing Ltd, Bristol

[20] Ohya K et al, 2009 Journal of Nuclear Materials 390-391 72 - 75

[21] Ohya K, 2011 private communication

[22] Kirschner A et al, 2011 J. Nucl. Mater. 415(1, Supplement) S239 - S245

[23] Airila M I et al, 2009 Physica Scripta 2009(T138) 014021

[24] Droste S et al, 2008 Plasma Physics and Controlled Fusion 50(1) 015006

[25] Aho-Mantila L et al, 2010 Contrib. Plasma Phys. 50(3-5) 439-444

[26] Strachan J et al, 2008 Nuclear Fusion 48(10) 105002

[27] Aho-Mantila L et al, 2011 38th EPS Conference on Plasma Physics P1.061 